\title{
Regulation of hypothalamic-pituitary-adrenal activity in response to cognitive therapy in patients with generalized anxiety disorder
}

\author{
GUSTAVO E. TAFET, DIEGO J. FEDER, DENISE P. ABULAFIA, and SILVIA S. ROFFMAN \\ Maimonides University, Buenos Aires, Argentina
}

\begin{abstract}
The aim of this study was to evaluate the efficacy of cognitive therapy (CT) in the treatment of generalized anxiety disorder (GAD), as it would be reflected through both psychological and psychoneuroendocrinological parameters. For this purpose, a group of outpatients with GAD were treated with CT for up to a maximum of 24 sessions. In order to assess psychological and biological changes, anxiety-related symptoms were evaluated according to the Hamilton Anxiety Rating Scale (HAM-A), and the hypothalamic-pituitary-adrenal (HPA) function was determined through assessment of circulating cortisol levels. A significant decrease in the HAM-A scores, along with significant changes in plasma cortisol levels, were observed after completion of treatment with CT. These observations contribute to demonstrate that the effect of certain psychotherapeutic approaches, such as CT, may be observed at both psychological and biological levels.
\end{abstract}

Psychosocial stress is a common condition in human life, a state of challenge or threat to which the organism, in order to preserve its internal equilibrium, reacts with an array of adaptive responses mediated by neural and neuroendocrine cascades. This involves the immediate release of catecholamines in the central nervous system and the autonomic nervous system and the activation of the limbic-hypothalamic-pituitary-adrenal (HPA) system, with the subsequent release of corticotropin-releasing hormone $(\mathrm{CRH})$, adrenocorticotropin hormone $(\mathrm{ACTH})$, and glucocorticoids, particularly cortisol (Akil \& Morano, 1995; Chrousos \& Gold, 1992). The particular features of this response will depend on the characteristics of the stressor itself, such as intensity and duration, and the individual psychological resources, which may determine its particular appraisal and the resulting coping strategies (Lazarus \& Folkman, 1984). Therefore, chronic stress, such as that observed during prolonged exposure to various stressful life events, along with the inadequacy to cope with them or the perceived loss of controllability, may lead to the persistent activation of the HPA system (Henry, 1992). Hence, the normal activity of the HPA axis, characterized by wide circadian variations with morning zeniths and evening nadirs, becomes altered during chronic stress (Halbreich, Asnis, Schindledecker, Zumoff, \& Nathan, 1985). This results in a sustained increase of cortisol levels (Ottenweller, Natelson, Pitman, \& Drastal, 1989), with a blunted circadian curve due to

Correspondence concerning this article should be addressed to G. E. Tafet, Department of Psychiatry and Neurosciences, Maimonides University, Hidalgo 775, Buenos Aires (1405), Argentina (e-mail: psychiatry@ maimonides.edu). increased levels in the evening and discrete decline in the morning (Chrousos \& Gold, 1998). This hyperactivation, with the resulting hypercortisolism, has long been associated with different clinical conditions, such as depression and anxiety disorders (Gold, Goodwin, \& Chrousos, 1988a, 1988b). The DSM-IV establishes different categories of anxiety disorders, including those characterized by a chronic course, such as the generalized anxiety disorder (GAD). This constitutes a highly prevalent condition, characterized by excessive anxiety and worry about various life events, implying an important source of subjective distress during a certain period of time (American Psychiatric Association, 1994). This may cause hyperactivation of the HPA axis, as we observed in a previous study in which a group of patients with GAD presented increased levels of circulating cortisol, similar to those observed in a group of patients with major depression (MDD; Tafet, Idoyaga-Vargas, et al., 2001).

It has been shown that patients with mood and anxiety disorders have been successfully treated with cognitive therapy (CT; Beck, Rush, Shaw, \& Emery, 1979), as it was described in different reports on the efficacy of CT in the treatment of depression and GAD (Borkovec \& Ruscio, 2001). It is likely that CT would be effective in the treatment of GAD not only by improving distressful clinical symptoms, but also by recovering psychoneuroendocrinological functions. Therefore, we hypothesized that improvement should be reflected not only by clinical assessment, but also by biochemical indicators of HPA function, such as the measurement of plasma cortisol levels. Hence, the present study was designed to evaluate the efficacy of $\mathrm{CT}$ in the treatment of a chronic anxiety disorder, such as GAD, as it would be reflected through both psychological and biochemical parameters. 


\section{METHOD}

The study subjects were patients admitted to the Stress Research Program, developed by the Department of Psychiatry and Neurosciences at Maimonides University. The protocol was evaluated and approved by the ethical committee of the University, and after the procedure had been fully explained to the subjects, written informed consent was obtained. The subjects were evaluated according to the Structured Clinical Interview for DSM-IV Axis I disorders (SCID; First, Spitzer, Gibbon, \& Williams, 1997) to establish the diagnostic categories and determine exclusionary diagnoses. Accordingly, all the patients included met the DSM-IV criteria for $\mathrm{GAD}$, and their severity of anxiety-related symptoms was assessed according to the Hamilton Anxiety Rating Scale (HAM-A). In addition, the severity of mood related symptoms was assessed according to the Hamilton Depression Rating Scale (HAM-D). Exclusion criteria were lifetime history of mania or psychosis and a history of substance abuse within the past 6 months. In addition, none of the subjects had been under treatment with antidepressants, neuroleptics, or glucocorticoids within the past 6 months prior to inclusion in or during the development of the study. Therefore, a total of 20 outpatients were recruited, 17 of whom ( 8 males and 9 females; ages, 24-58 years, $M=42.4$ ) completed the treatment. From the initial 20 outpatients recruited for the study, 3 subjects ( 1 male and 2 females) additionally received antidepressants and, therefore, were excluded from the protocol. In addition, a group of 8 volunteers ( 4 males and 4 females), recruited among outpatients from other departments, also met $D S M-I V$ criteria for GAD but did not receive any psychotherapeutic nor psychopharmacologic treatment during the time of the study and did not met any of the exclusion criteria, therefore constituting a control group.

\section{HPA Assessment}

Two peripheral blood samples were drawn from each subject at 0800 and 1600 hours of the same day, at the beginning of the study, in the laboratory of Dr. J. Glatstein at Maimonides University. Plasma cortisol concentrations were determined by chemiluminescence with the use of a standard, commercially available competitive immunoassay (Diagnostic Products Corp.). The normal range was taken as 7-25 $\mathrm{ng} / \mathrm{dl}$ for the morning and 2-9 $\mathrm{ng} / \mathrm{dl}$ for the afternoon (Sapse, 1997). Two additional peripheral blood samples were drawn from each subject at 0800 and 1600 hours of the same day in order to assess plasma concentrations of cortisol after completion of their treatment with $\mathrm{CT}$, or after 24 weeks in the control group.

\section{Treatment}

The patients began treatments with CT as soon as they were interviewed and diagnosed, receiving one 45-min individual therapy session, once a week during 24 weeks, up to a maximum of 24 sessions. Treatments were conducted by experienced therapists of the Department of Psychiatry and Neurosciences at Maimonides University. Positive response to treatment was defined as a reduction in the HAM-A score of $50 \%$ or more.

\section{Statistical Analysis}

In order to test for differences in cortisol levels, in the morning and afternoon, and in HAM-A scores, a multivariate analysis of variance was carried out, with cortisol values after and before treatment and clinical assessment after and before treatment as dependent variables.

\section{RESULTS}

From a total of the 20 outpatients recruited, 17 subjects ( 8 males and 9 females; ages, 24-58 years, $M=$
42.4) completed the study. All the patients included met the $D S M-I V$ criteria for GAD, and the severity of anxietyrelated symptoms was assessed according to the HAM-A. Analogously, the severity of mood-related symptoms was assessed according to the HAM-D. Out of the original sample, a group of 3 patients met the criteria for both GAD and MDD and, according to the severity of their depressive symptoms (HAM-D score $=25.3, S D=$ 3.9), needed to be treated with antidepressants in addition to CT; these patients, therefore, were excluded from the protocol. All the remaining 17 GAD patients presented only mild symptoms of depression (HAM-D score $<14$ ).

Cortisol concentrations in plasma were determined in the morning (0800 hours) and afternoon (1600 hours), as described previously. Regarding cortisol levels in the morning, we observed normal values in the group of treated patients at the beginning $(M=20.3, S D=8.2)$, and no significant changes were observed after 24 sessions of CT $[M=18.3, S D=6.6 ; F(1)=1.5$, n.s. $]$. The comparison group also presented normal values at the beginning $(M=23.1, S D=5)$, and no significant changes were observed after 24 weeks $[M=21.6, S D=5.2$; $F(1)=1.4$, n.s.]. Regarding cortisol in the afternoon, we observed increased levels before treatment $(M=14.6$, $S D=3.4)$ and a decrease with significant differences from those registered after treatment $[M=7.9, S D=$ $1.1 ; F(1)=26.1, p<.0001$; Figure 1]. The comparison group also presented increased levels at the beginning $(M=16.5, S D=3.3)$ but no significant changes after 24 weeks without treatment $[M=14.5, S D=4 ; F(1)=$ $19.8, p<.01]$. Analogously, HAM-A scores were also

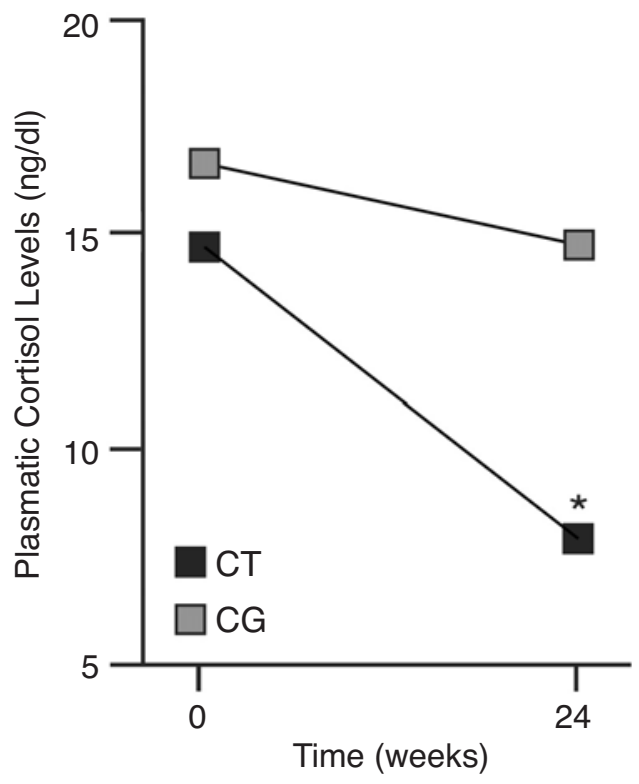

Figure 1. Assessment of plasma cortisol concentrations in the afternoon (1600 hours), at the beginning and after 24 weeks, for patients with generalized anxiety disorder (GAD) treated with cognitive therapy (CT) and for a control group (CG) with GAD without treatment. * $p<.00001$. 
determined before the beginning and after the completion of the treatment, as described previously. Hence, the patients with GAD treated with CT presented high scores at the beginning $(M=29.5, S D=5.2)$, which decreased in a significant manner after completion of treatments 24 weeks later $[M=10.0, S D=4.0 ; F(1)=126.9, p<$ .00001 ; Figure 2]. The subjects with GAD who belonged to the comparison group presented high scores at the beginning $(M=30.6, S D=3.4)$ and also after 24 weeks without treatment $[M=23.7, S D=5.9 ; F(1)=20.2$, $p<.001]$.

\section{DISCUSSION}

There is ample evidence supporting the efficacy of different psychotherapeutic approaches in the treatment of mood and anxiety disorders. Among these approaches, CT has been shown to be particularly effective, alone or in combination with pharmacotherapy, in the treatment of mood and different anxiety disorders, such as GAD (Borkovec \& Ruscio, 2001; Gorman, 2002).

Regarding biological parameters, it has been shown that patients with mood and anxiety disorders often present dysregulation of the HPA axis, which may be reflected in a sustained increase of cortisol levels, with a blunted circadian curve due to increased levels in the evening. This hyperactivation, with the resulting hypercortisolism, has long been associated with different clini-

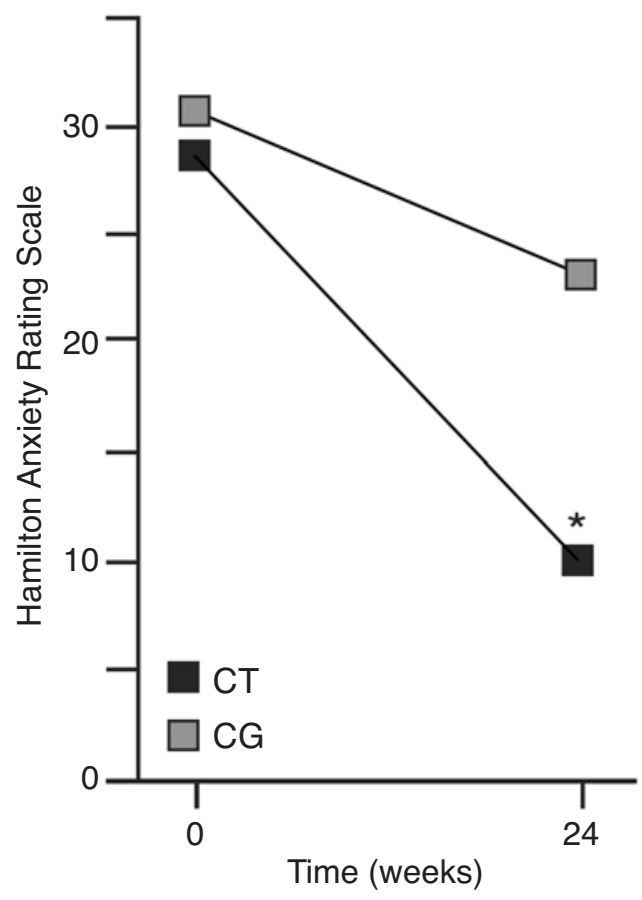

Figure 2. Anxiety-related symptoms assessed according to the Hamilton Anxiety Rating Scale, at the beginning and after 24 weeks, for patients with generalized anxiety disorder (GAD) treated with cognitive therapy (CT) and for a control group (CG) with GAD without treatment. ${ }^{*} p<.00001$. cal conditions, such as mood and anxiety disorders (Gold et al., 1988a, 1988b). In this regard, it has been proposed that increased levels of cortisol could be involved in the molecular mechanisms that link both clinical conditions (Holsboer, 2000; Tafet, Toister-Achituv, \& Shinitzky, 2001).

In the present study, we observed a significant decrease in previously increased levels of circulating cortisol, along with a significant decrease in HAM-A scores, reflecting a clinical improvement in response to CT in patients with GAD. The fact that patients with GAD who also presented severe symptoms of depression, reflected in high HAM-D scores, were excluded from the study and those who were included presented only mild symptoms of depression, reflected in low HAM-D scores, strongly suggests that the observed changes were associated with the effect of CT on more prominent anxietyrelated symptoms, rather than with the effect on the mild associated symptoms. It has been proposed that the CT treatment of GAD is based on a cognitive model of vulnerability (Beck, 1976) reflected in a balance between perceived threats and resources. The positive effects produced by CT at the clinical level could be understood as a significant improvement in the appraisal of this process. This includes the subjective perception of enhanced ways of dealing with uncertainty, the remodeling of underlying dysfunctional beliefs, and the development of more effective strategies aimed at identifying and controlling worry (Butler, 1994). In this regard, it has been proposed that a central feature of worry is its uncontrollability, further suggesting that uncontrollability of worry is a central feature of GAD (Wells \& Butler, 1997). Therefore, the positive response observed in GAD patients treated with CT could be understood as a result of improved controllability, and this could be developed from a more adaptive appraisal and the resulting more effective coping strategies.

The hypothesis raised in the study presented is in line with the notion that uncontrollable situations of distress may be associated with a dysregulation of the HPA axis and is coherent with the cognitive model mentioned. Our observation that patients with GAD presented significant improvement at both psychological and biological levels after treatment with CT exclusively may represent a potential link between both conceptions and further suggests that there should be more extensive research on the effects of psychotherapy at the clinical and the psychoneuroendocrinological levels.

\section{CONCLUSION}

Our observation that patients with GAD, who presented increased levels of circulating cortisol, decreased their cortisolemia along with their clinical improvement after treatment with CT constitutes an indirect demonstration of the effect of a psychotherapeutic approach through both clinical and biological parameters. Since patients with GAD have been reported to be successfully 
treated with CT when it was used as a monotherapy or in combination with pharmacotherapy, CT could provide a potential strategy not only to successfully treat chronic anxiety disorders, such as GAD, but also to prevent the development of more severe conditions, such as depression, also associated with dysregulation of the HPA axis.

\section{REFERENCES}

Akil, H. A., \& Morano, M. I. (1995). Stress. In F. E. Bloom \& D. J. Kupfer (Eds.), Psychopharmacology: The fourth generation of progress (pp. 773-785). New York: Raven.

American Psychiatric Association (1994). Diagnostic and statistical manual of mental disorders: DSM-IV (4th ed., pp. 393-444). Washington, DC: Author.

Beck, A. T. (1976). Cognitive therapy and the emotional disorders. New York: International Universities Press.

Beck, A. T., Rush, A. J., Shaw, B. F., \& Emery, G. (1979). Cognitive therapy of depression. New York: Guilford.

Borkovec, T. D., \& Ruscio, A. M. (2001). Psychotherapy for generalized anxiety disorder. Journal of Clinical Psychiatry, 62(Suppl. 11), $37-42$.

BUTLER, G. (1994). Treatment of worry in generalised anxiety disorder. In G. C. L. Davey \& F. Tallis (Eds.), Worrying: Perspectives on theory, assessment, and treatment (pp. 209-228). Chichester, U.K.: Wiley.

Chrousos, G. P., \& Gold, P. W. (1992). The concepts of stress and stress system disorders: Overview of physical and behavioral homeostasis. Journal of the American Medical Association, 267, 12441252.

Chrousos, G. P., \& Gold, P. W. (1998). A healthy body in a healthy mind - and vice versa-The damaging power of "uncontrollable" stress. Journal of Clinical Endocrinology \& Metabolism, 83, 18421845 .

First, M. B., Sitzer, R. L., Gibbon, M., \& Williams, J. B. W. (1997). Structured clinical interview for DSM-IV Axis I disorders, clinician version ( $S C I D-C V$ ). Washington, DC: American Psychiatric Publishing.

Gold, P. W., Goodwin, F. K., \& Chrousos, G. P. (1988a). Clinical and biochemical manifestations of depression. Relation to the neurobiology of stress (1). New England Journal of Medicine, 319, 348-353.

Gold, P. W., Goodwin, F. K., \& Chrousos, G. P. (1988b). Clinical and biochemical manifestations of depression. Relation to the neurobiology of stress (2). New England Journal of Medicine, 319, 413-420.

GoRMAN, J. M. (2002). Treating generalized anxiety disorder. Journal of Clinical Psychiatry, 63(Suppl. 8), 17-23.

Halbreich, U., Asnis, G. M., Schindendecker, R., Zumoff, B., \& Nathan, R. S. (1985). Cortisol secretion in endogenous depression: I. Basal plasma levels. Archives of General Psychiatry, 42, 904-908.

Henry, J. P. (1992). Biological basis of the stress response. Integrative Physiological \& Behavioral Science, 27, 66-83.

HOLSBOER, F. (2000). The corticosteroid receptor hypothesis of depression. Neuropsychopharmacology, 23, 477-501.

Lazarus, R. S., \& FolKman, S. (1984). Stress, appraisal, and coping. New York: Springer-Verlag.

Ottenweller, J. E., Natelson, B. H., Pitman, D. L., \& Drastal, S. D. (1989). Adrenocortical and behavioral responses to repeated stressors: Toward an animal model of chronic stress and stress-related mental illness. Biological Psychiatry, 26, 829-841.

SAPSE, A. T. (1997). Cortisol, high cortisol diseases and anti-cortisol therapy. Psychoneuroendocrinology, 22(Suppl. 1), S3-S10.

Tafet, G. E., Idoyaga-Vargas, V. P., Abulafia, D. P., Calandria, J. M., Roffman, S. S., Chiovetta, A., \& Shinitzky, M. (2001). Correlation between cortisol level and serotonin uptake in patients with chronic stress and depression. Cognitive, Affective, \& Behavioral Neuroscience, 1, 388-393.

TAFet, G. E., ToIster-Achituv, M., \& Shinitzky, M. (2001). Enhancement of serotonin uptake by cortisol: A possible link between stress and depression. Cognitive, Affective, \& Behavioral Neuroscience, 1, 96-104.

WELLS, A., \& BUTLER, G. (1997). Generalized anxiety disorder. In D. M. Clark \& C. G. Fairburn (Eds.), Science and practice of cognitive behaviour therapy (pp. 155-178). Oxford: Oxford University Press.

(Manuscript received November 26, 2003; revision accepted for publication October 20, 2004.) 